\title{
Consultation-liaison Psychiatry: An Urgent Yet Unmet Need in Our Country
}

Psychiatry has come a long way from asylum-based treatments to general hospital psychiatry units (GHPU). Although setting up of GHPUs has led to widespread collaboration between psychiatrists and other medical and surgical specialists, role of psychiatrists is still limited to providing consultation in cases admitted in medical and surgical wards which develop psychiatric complications or have a diagnosed psychiatric illness. There is no separate unit or department specifically dedicated to "Consultation-liaison Psychiatry" in terms of teaching or research. The scenario in private hospitals is even worse. In many cases, psychiatric complications and comorbidities are ignored or "suppressed" by the treating physicians (often at the request of family members of patients) in apprehension that

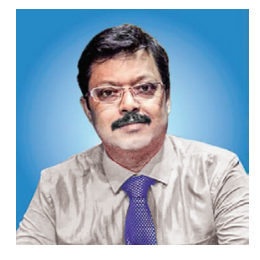
the insurance company will "reject" their claim. Thankfully, the Mental Healthcare Act, 2017, mandates that the insurance companies should have provisions for covering the cost of treatment of the mentally ill. The Insurance Regulatory Authority of India has also made certain initiatives in this regard. Hopefully, this step would bolster the utilization of psychiatric services in private hospitals and nursing homes as well. Overall, an effort is seriously needed to boost "Consultation-liaison Psychiatry" in India across government and private healthcare facilities for betterment of patients in general and growth and de-stigmatization of psychiatry.

Gautam Saha

Director, Clinic Brain, Neuropsychiatric Institute and Research Center Barasat, West Bengal, India e-mail:drgautamsaha@gmail.com 